\title{
Tamoxifen and raloxifene modulate gap junction coupling during early phases of retinoic acid-dependent neuronal differentiation of NTera2/D1 cells
}

\author{
Liane Dahm • Fanny Klugmann • \\ Angeles Gonzalez-Algaba • Bernhard Reuss
}

Received: 3 December 2009 / Accepted: 12 April 2010/Published online: 1 May 2010

(C) The Author(s) 2010. This article is published with open access at Springerlink.com

\begin{abstract}
Gap junctions (GJ) represent a cellular communication system known to influence neuronal differentiation and survival. To assess a putative role of this system for neural effects of tamoxifen (TAM) and raloxifene (RAL), we used the human teratocarcinoma cell line NTera2/D1, retinoic acid (RA)-dependent neuronal differentiation of which is regulated by gap junctions formed of connexin43 (Cx43). As demonstrated by Western blot analysis, concentrations above $1 \mu \mathrm{mol} / 1$ for TAM, and $0.1 \mu \mathrm{mol} / 1$ for RAL lead to a temporary time- and concentration-dependent increase in Cx43 immunoreactivity, which reached a peak for TAM after 1 day and for RAL after 2 days. Immunocytochemical stainings revealed the increase in $\mathrm{Cx} 43$ immunoreactivity to result from an accumulation in intracellular compartments such as the Golgi apparatus or lysosomes. In addition, TAM and RAL were able to prevent the RA-dependent decrease of $\mathrm{Cx} 43$ immunoreactivity in NTera2/D1 cells, normally observed during neuronal differentiation. This suggested a suppression of neuronal differentiation to result from these sub-
\end{abstract}

L. Dahm · F. Klugmann • A. Gonzalez-Algaba •

B. Reuss $(\bowtie)$

Center for Anatomy-Neuroanatomy,

University of Göttingen,

Kreuzbergring 36,

37075 Göttingen, Germany

e-mail: breuss@gwdg.de stances. According to this, treatment of NTera2/D1 cells with $10 \mu \mathrm{mol} / 1 \mathrm{TAM}$ or RAL during weeks 1 and 2 of a 6 weeks RA-driven differentiation schedule impaired, whereas treatment during weeks 5 and 6 did not impair, neuronal differentiation of these cells. Modulation of GJ coupling between NTera2/D1 cells by TAM and RAL seems therefore to perturb early neuronal differentiation, whereas differentiated neurons in the mature brain seem to be not affected. These effects could be of importance for actions of TAM and RAL on early embryonic steps of nervous system formation.

Keywords Connexin43 - Gap junction · Neuron · NTera2/D1 · Raloxifene · Tamoxifen · Retinoic acid

$\begin{array}{ll}\text { Abbreviations } \\ \text { Cx43 } & \text { Connexin43 } \\ \text { DMSO } & \text { Dimethylsulfoxide } \\ \text { ECL } & \text { Enhanced chemiluminescence } \\ \text { ER } & \text { Estrogen receptor } \\ \text { FITC } & \text { Fluorescein-isothiocyanate } \\ \text { GJ } & \text { Gap junctions } \\ \text { LY } & \text { Lucifer yellow } \\ \text { PBS } & \text { Phosphate-buffered saline } \\ \text { PFA } & \text { Paraformaldehyde } \\ \text { RA } & \text { Retinoic acid } \\ \text { RAL } & \text { Raloxifene } \\ \text { SDS } & \text { Sodium dodecyl sulfate } \\ \text { SERMs } & \text { Selective estrogen receptor modulators } \\ \text { TAM } & \text { Tamoxifen }\end{array}$


TBS TRIS-buffered saline

TRIS Tris(hydroxymethyl)-aminomethane

\section{Introduction}

Tamoxifen (TAM) and raloxifene (RAL) are nonsteroidal selective estrogen receptor modulators widely used for the treatment of estrogen responsive breast cancer as well as for breast cancer prophylaxis in women at high risk of developing this disease (Wickerham et al. 2009). Both drugs are also used to prevent osteoporosis in postmenopausal women (Reginster and Devogelaer 2006), and high-dose treatment with TAM in combination with radiation therapy is able to inhibit proliferation in glioblastoma cells (da Rocha et al. 1999). Besides their specific actions on bone and tumor cells, both substances are known to affect also other organ systems such as liver (Williams et al. 1993), endometrium (Fisher et al. 1994), and the brain (Eberling et al. 2004; Yaffe et al. 2005; Palmer et al. 2008; Collins et al. 2009). In addition, TAM has been shown to also affect fetal development, since prenatal exposure results in craniofacial malformations as well as deviations in the female reproductive tract (Berger and Clericuzio 2008; Cunha et al. 1987). Also high doses of RAL are known to elicit teratogenic effects resulting in reduced fetal growth and organ malformations, i.e., of heart and kidney (Byrd and Francis 1998). Also impaired neurogenesis has been demonstrated for TAM, since in rats treated with TAM during late fetal and early postnatal phases of development revealed impaired hippocampal morphology (Nobakht et al. 2009). The present study was designed to clarify in vitro, whether TAM and RAL affect also neuronal differentiation of human-derived cells and if so, whether intercellular communication by gap junctions (GJ) could be involved in these processes.

GJ provide a communication pathway with a wellknown impact on cell differentiation and survival during development and in the adult organism (Revel 1986; Guthrie and Gilula 1989; Wei et al. 2004). GJ are assemblies of membrane channels connecting the cytoplasm of neighboring cells. A functional GJ channel is thereby established by docking of two hemichannels, provided by each of the adjacent cells. Every hemichannel consists of a hexagonal array of six connexin $(\mathrm{Cx})$ proteins, surrounding a central channel pore, allowing the propagation of second messengers, nutrients, and other metabolic products with molecular weights of less than $1 \mathrm{kDa}$ (Meșe et al. 2007). Cx belong to a family of more than $20 \mathrm{GJ}$ proteins, which differ with regard to functional parameters like permeability and open probability, and are expressed in a tissue and cell-type-specific manner (Söhl and Willecke 2004). Functionality of GJ channels is regulated by different ways, including changes in expression levels or levels of membrane integration as well as epigenetic modifications such as a step-wise phosphorylation (Solan and Lampe 2009). During neuronal differentiation, GJ coupling reveals a progressive decrease from extensive coupling between early neuronal precursor cells, towards a more restricted coupling pattern or an entire lack of coupling between differentiated neurons (Sutor and Hagerty 2005; Bruzzone and Dermietzel 2006). The corresponding GJ protein is $\mathrm{Cx} 43$, which is highly expressed in neuronal precursor cells and gradually decreases during progredient neuronal differentiation.

A cell culture model for neuronal differentiation is NTera2/D1 cells, a line of human teratocarcinoma cells, derived from a lung metastasis of a testicular germ cell tumor (Andrews 1984). NTera2/D1 cells share some common properties with early embryonic stem cells, and can be differentiated into neurons and glial cells by treatment with retinoic acid (RA; Pleasure and Lee 1993). Undifferentiated NTera2/D1 cells are coupled by GJ, and as an early event during RA-induced neuronal differentiation, expression of $\mathrm{Cx} 43$, the corresponding GJ protein, is diminished (Bani-Yaghoub et al. 1997; Boucher and Bennett 2003). On the other hand, inhibition of GJ coupling between NTera2/D1 cells has also been shown to abolish their RA-dependent neuronal differentiation (Bani-Yaghoub et al. 1999). Therefore, GJ are first of major importance for the induction of early neuronal differentiation of NTera2/D1 cells, and are later downregulated during their neuronal differentiation. NTera2/D1 cells have already earlier been used to test neurotoxicity of several substances with different levels of embryotoxicity such as acrylamide, lithium, valproic acid, and hydroxyurea, and were found to detect neurotoxic substances in a highly reliable manner (Woehrling et al. 2007, 2010; Hill et al. 2008). Due to these properties and to the central role of GJ-mediated intercellular 
communication for their neuronal differentiation, NTera2/D1 cells were chosen here as a model system to address the question for a putative role of gap junctions in mediating effects of TAM and RAL on early neuronal differentiation.

\section{Materials and methods}

Cell culture and neuronal differentiation of NTera2/D1 cells

NTera2/D1 cells were obtained from the German Collection for microorganisms and cell cultures (http:// www.dsmz.de). Cells were seeded in Dulbecco's modified Eagle's medium, supplemented with $10 \%$ $\alpha$-minimal essential medium ( $\alpha$-MeM), 10\% fetal bovine serum, $100 \mathrm{U} / \mathrm{ml}$ penicillin, $100 \mu \mathrm{g} / \mathrm{ml}$ streptomycin, $2 \mathrm{mmol} / \mathrm{l} \mathrm{L}$-glutamine, and $1 \mathrm{mmol} / \mathrm{l}$ sodium pyruvate (all from http://www.invitrogen.com) on standard polystyrene cell culture flasks (http://www. sarstedt.com), with the flasks being maintained in a humidified incubator at $37^{\circ} \mathrm{C}$ under $5 \% \mathrm{CO}_{2}$. Once a week, subconfluent monolayers of NTera2/D1 cells were split by incubation with Trypsin-EDTA (http:// www.invitrogen.com), and 100,000 cells were seeded on cell culture flasks for maintenance culture. Of the remaining cells, 2 million were seeded either on sixwell plates for protein extraction, or on 12-mm coverslips in a 24-well plate for immunocytochemistry. For scrape loading, cells were plated at the same density on 100-mm cell culture grade Petri dishes (www.sarstedt.com). Stock solutions of either TAM, RAL, or RA were prepared in cell culture grade dimethylsulfoxide (DMSO, http://www.merck.com) and dissolved in cell culture medium at concentrations as indicated in the text. All controls were supplied with the corresponding amount of vehicle (DMSO, http:// www.merck.com). In order to minimize differences in cell density, cells were treated according to the following schedule: for a 1-day treatment, cells were exposed at day 6 , for a 2 -days treatment at day 5 , and for a 4-day treatment at day 3 and 5 after plating. All cells were harvested or fixed on day 7 . For neuronal differentiation, 2 million of NTera2/D1 cells were seeded on 12-mm coverslips in a 24-well plate followed by 6 weeks treatment with $10 \mu \mathrm{mol} / \mathrm{l} \mathrm{RA}$. To test for early and late effects of TAM and RAL on neuronal differentiation, cells were additionally treated with $10 \mu \mathrm{mol} / 1$ of TAM or RAL either during weeks 1 and 2 or weeks 5 and 6 of a 6-week differentiation schedule.

\section{Western blot analysis}

Ten micrograms of total cellular protein dissolved in electrophoresis sample buffer $(0.5 \mathrm{mmol} / 1 \mathrm{TRIS} / \mathrm{HCl}$, pH 6.8, 2\% sodium dodecyl sulfate (SDS) $(w / v), 10 \%$ glycerol $(v / v), 5 \% \quad \beta$-mercaptoethanol $(v / v)$, and $0.001 \%$ bromophenol blue $(w / v))$, was size fractionated by $8.5 \%$ SDS-polyacrylamide gel electrophoresis. Proteins were blotted onto polyvinylidene difluoride membranes (http://www.carl-roth.de), which were then blocked for $1 \mathrm{~h}$ at $4^{\circ} \mathrm{C}$ in $3 \%(w / v)$ nonfat dry milk dissolved in TRIS-buffered saline (TBS). Blots were probed with antibodies directed to either $\mathrm{Cx} 43$ (rabbit polyclonal, dilution 1:2,500; http://www.invitrogen. com), or $\beta$ III-tubulin (rabbit polyclonal, 1:2,500; $\mathrm{http}: /$ www.sigmaaldrich.com), diluted in TBS with $3 \%$ nonfat dry milk. After washing, primary antibodies were detected by incubation with a peroxidaseconjugated goat anti-rabbit secondary antibody (dilution 1:20,000; http://www.piercenet.com), which was visualized by exposing CLXposure X-ray films (http:// www.piercenet.com), during the application of an enhanced chemiluminescence (ECL) detection solution (ECL Western Blotting Substrate; http://www.pierce net.com). After development and fixation, the films were scanned, and the ECL-signals were quantified densitometrically, using the Metamorph image analysis software (www.moleculardevices.com). For loading control, blots were stripped for $15 \mathrm{~min}$ in $0.1 \mathrm{~mol} / 1$ $\mathrm{NaOH}$, and reprobed with an antibody directed to $\beta$ actin (mouse monoclonal, dilution 1:5,000; http:// www.sigmaaldrich.com). Detection was performed as described above, using a peroxidase-conjugated rabbit anti-mouse secondary antibody (dilution 1:20,000; http://www.piercenet.com). Mean values and standard deviations of the signal intensities of four independent experiments were calculated and tested for significance by Student's two-tailed $t$ test.

Immunocytochemistry

Cells on 12-mm glass cover slips were fixed for $10 \mathrm{~min}$ by $4 \%$ paraformaldehyde (PFA) in phosphate-buffered saline (PBS). After washing and permeabilization with PBS/Tween-20 they were blocked with $0.5 \%$ goat 
serum in PBS/Tween-20, and incubated over night at $4^{\circ} \mathrm{C}$ with primary antibodies directed to either $\mathrm{Cx} 43$ (rabbit polyclonal, 1:400; http://www.invitrogen.com) or $\beta$ III-tubulin (rabbit polyclonal, 1:100; http://www. sigmaaldrich.com). For visualization, fixed cells were incubated sequentially with a biotinylated goat antirabbit secondary antibody (dilution 1:400; http://www. sigmaaldrich.com), followed by a complex of fluorescein-isothiocyanate and streptavidin (dilution 1:400; http://www.sigmaaldrich.com). Cover slips were then mounted on glass slides using an aqueous fluorescence mounting medium (http://www.dako. com). Microscopic evaluation was performed with an Axiophot microscope (http://www.zeiss.com) equipped with epifluorescence, at standardized illumination conditions, and camera settings.

\section{Scrape loading}

For scrape loading, confluent monolayers of NTera2/D1 cells treated as indicated in the text, were washed three times with sterile saline $(0.9 \% \mathrm{NaCl})$, and were covered with $1 \mathrm{mg} / \mathrm{ml}$ Lucifer yellow (LY) $\mathrm{CH}$ (http://www. sigmaaldrich.com) in sterile saline at $37^{\circ} \mathrm{C}$. To start dye loading, four cuts were set in the monolayer with a scalpel blade. After $2 \mathrm{~min}$ the dye solution was removed, followed by three washing steps using sterile saline. Four minutes after setting the scrapes, dye spreading was stopped by fixing the cells for $10 \mathrm{~min}$ with an ice-cold solution of 1\% PFA in sterile saline. After three washes with sterile saline, dye spreading was documented photographically, using an inverse microscope equipped with epifluorescence (http:// www.zeiss.com). Again, all illumination conditions and camera settings were standardized. For statistical evaluation, distances of dye spreading were measured at 10 locations in each of the four scrapes in four independently treated Petri dishes $(n=40)$. Significance was analyzed by Student's two-tailed $t$ test.

\section{Results}

Both TAM and RAL cause a temporary upregulation of Cx43 in NTera2/D1 cells and a shift in subcellular localization

Phase-contrast images of NTera2/D1 cells treated for 2 days with either TAM or RAL alone (Fig. 1a-c) or in combination with RA (Fig. 1d-f) demonstrate that neither of the treatments elicits gross morphological changes in NTera2/D1 cells compared to vehicletreated control cultures.

In contrast to this, Western blot analysis reveals that TAM leads to a temporary upregulation of total Cx43 immunoreactivity in NTera2/D1 cells with a maximal intensity after 1 day of treatment (Fig. 2a). This upregulation is concentration dependent resulting in a significant induction only in cells treated with TAM at concentrations of $1 \mu \mathrm{mol} / \mathrm{l}$ and higher (Fig. 2b, c). With regard to phosphorylation, no signficant changes in the relative distribution of phosphorylated and unphosphorylated forms of Cx43 can be found (Fig. 2b).

For RAL Western blot analysis reveals also a temporary upregulation of total $\mathrm{Cx} 43$ immunoreactivity in NTera2/D1 cells; however, for this substance maximal intensity is reached after 2 days of treatment (Fig. 3a). This upregulation is also concentration dependent resulting in a significant induction in cells treated with RAL at concentrations of $0.1 \mu \mathrm{mol} / 1$ and higher (Fig. 3b, c). Also for RAL, no significant changes in the relative distribution of phosphorylated and unphosphorylated forms of $\mathrm{Cx} 43$ can be found (Fig. 3b).

Similar results for $\mathrm{Cx} 43$ immunoreactivity were obtained by immunofluorescent staining (Fig. 4a-f) revealing a distinct increase of staining intensity in NTera2/D1 cells by both TAM (Fig. $4 \mathrm{a}-\mathrm{c}$ ) and RAL (Fig. 4d-f). In addition, a subcellular shift of $\mathrm{Cx} 43$ immunoreactivity can be observed with mostly peripheral localization in untreated cells, probably representing the cell membranes (Fig. $4 \mathrm{a}, \mathrm{d}$ ), and a predominant intracellular localization in cells treated with either TAM (Fig. 4b, c) or RAL (Fig. 4e, f).

TAM and RAL prevent the RA-dependent decrease of $\mathrm{Cx} 43$ in NTera2/D1 cells

The upregulation of $\mathrm{Cx} 43$ immunoreactivity by TAM and RAL in NTera2/D1 cells raises the question, whether these substances are able to prevent the RA-dependent reduction in $\mathrm{Cx} 43$ during neuronal differentiation described earlier for these cells (Bani-Yaghoub et al. 1997; Boucher and Bennett 2003). In order to answer this question, NTera2/D1 cells were cotreated with $10 \mu \mathrm{mol} / 1 \mathrm{RA}$ together with either TAM (Fig. 5a, b), or RAL 

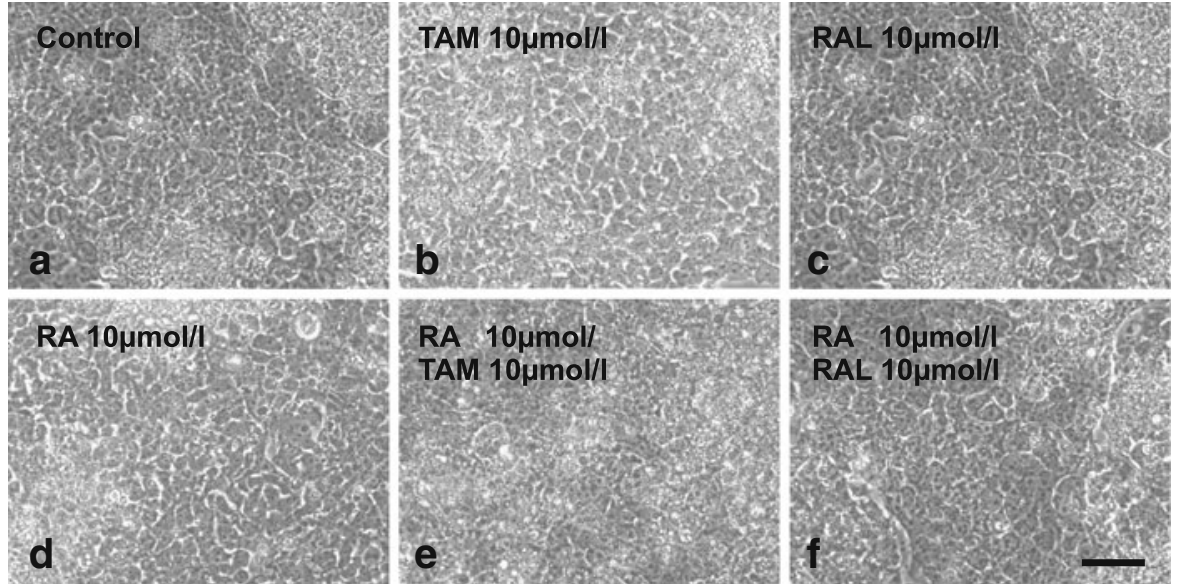

Fig. 1 Phase contrast images of NTera2/D1 cells treated for 2 days with either TAM or RAL alone or in combination with RA. a-c Cells treated with vehicle (DMSO) alone (a), or with $10 \mu \mathrm{mol} / 1 \mathrm{TAM}$ (b) or RAL (c). d-f Cultures treated with

(Fig. 5c) at concentrations ranging from 0.010 to $10 \mu \mathrm{mol} / \mathrm{l}$.

As Western blot analysis demonstrates, only the highest concentration of $10 \mu \mathrm{mol} / \mathrm{l} \mathrm{TAM}$ is able to significantly diminish RA-dependent downregulation of Cx43 in NTera2/D1 cells (Fig. 5a, b). In contrast to this RAL is much more efficient in preventing the RA-dependent decrease of $\mathrm{Cx} 43$ immunoreactivity in NTera2/D1 cells, leading already at a concentration of $0.1 \mu \mathrm{mol} / 1$ to a significant attenuation of the RAdependent downregulation of $\mathrm{Cx} 43$ (Fig. 5c).

The results obtained by Western blot analysis are confirmed by immunocytochemistry (Fig. 6a-f), where cotreatment of cells with RA together with either TAM (Fig. 6b, c) or RAL (Fig. 6e, f) is able to induce a distinct increase in total $\mathrm{Cx} 43$ immunoreactivity as compared to cells treated with RA alone (Fig. 6a, d). As in the single treatments, both TAM and RAL lead to a shift in subcellular distribution of Cx43 immunoreactivity from a predominant membrane-associated localization (Fig. 6a, d) to a preponderant staining in cytoplasmic organelles (Fig. 6b, c, e, f).

TAM and RAL alone or together with RA lead also to changes of functional GJ coupling in NTera2/D1 cells

To clarify whether the changes in $\mathrm{Cx} 43$ expression described above lead also to altered functional GJ
$10 \mu \mathrm{mol} / 1 \mathrm{RA}$ alone (d) or together with $10 \mu \mathrm{mol} / 1 \mathrm{TAM}$ (e) or RAL (f). No gross morphological changes can be observed. Scale bar represents $50 \mu \mathrm{m}$

coupling, we investigated the effects of TAM and RAL alone or in combination with RA on spreading of the GJ permeant dye LY. As revealed by scrape loading (Fig. 7a-f), treatment with $10 \mu \mathrm{mol} / 1 \mathrm{TAM}$ leads to a significant increase in the spreading distance of LY in NTera2 D1 cells, compared to untreated controls (Fig. 7a, b, and e). In contrast, treatment with $10 \mu \mathrm{mol} / \mathrm{l} \mathrm{RA}$ results in a significant reduction of dye spreading (Fig. 7c and e), which cannot be observed in cells simultaneously treated with $10 \mu \mathrm{mol} / 1$ of both RA and TAM (Fig. $7 \mathrm{~d}$ and e). Similar results were obtained for RAL (Fig. 7f), for which substance a treatment at a concentration of $10 \mu \mathrm{mol} / \mathrm{l}$ leads to an induction of dye spreading between NTera2/D1 cells, whereas $10 \mu \mathrm{mol} / \mathrm{l}$ RA blocks functional GJ coupling. Likewise cells treated with $10 \mu \mathrm{mol} / 1 \mathrm{RA}$ together with $10 \mu \mathrm{mol} / 1 \mathrm{RAL}$ do not reveal a blockade of LY transfer between NTera2/ D1 cells (Fig. 7f).

Treatment of NTera2/D1 cells with TAM and RAL during early phases of RA-dependent neural differentiation leads to impaired neuron formation

In a final set of experiments the impact of changes in GJ expression and function caused by TAM and RAL on RA-induced neuronal differentiation of NTera2/D1 cells was analyzed by immunocytochemical detection of the neuronal marker protein $\beta$ III-Tubulin (Fig. 8a-h). Cotreatment of NTera2/D1 cells during the first 

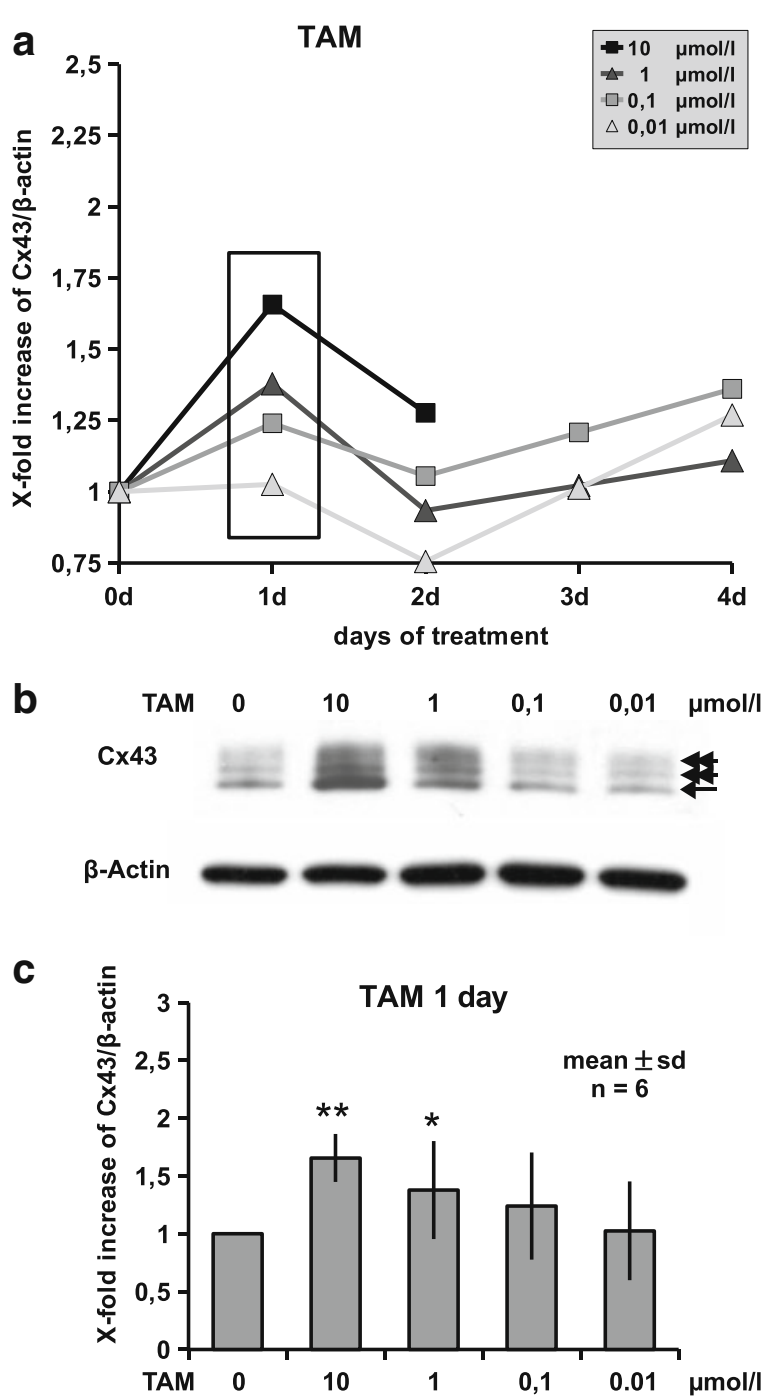

Fig. 2 Time and concentration dependency of TAM effects on Cx43 immunoreactivity in NTera2/D1 cells as revealed by Western blot analysis. a Densitometric evaluation of a series of Western blots demonstrates that in undifferentiated NTera2/D1 cells, TAM leads to a time- and concentration-dependent increase in $\mathrm{Cx} 43$ immunoreactivity, reaching a maximum after a 1 day treatment. b Representative Western blot of $\mathrm{Cx} 43$ immunoreactivity in NTera2/D1 cells, treated for 1 day with TAM at concentrations between 10 and $0.01 \mu \mathrm{mol} / 1$, as depicted by the square in (a). c Diagram of the statistical evaluation of a series of blots as shown in (b), demonstrating a significant upregulation of $\mathrm{Cx} 43$ immunoreactivity by TAM at concentrations of 10 and $1 \mu \mathrm{mol} / 1 .{ }^{*} p \leq 0.05 ; * * p \leq 0.01$

2 weeks of a 6 weeks RA-induced differentiation schedule with TAM (Fig. 8a, b, and g) or RAL (Fig. 8d, e, and h) results in a neuronal differentiation defect with significantly reduced numbers of neuron clusters and diminished neuronal process formation. In contrast to this, cotreatment with TAM (Fig. 8c and g) or RAL (Fig. $8 \mathrm{f}$ and $\mathrm{h}$ ) during the last 2 weeks of a 6 weeks RA-induced differentiation schedule has

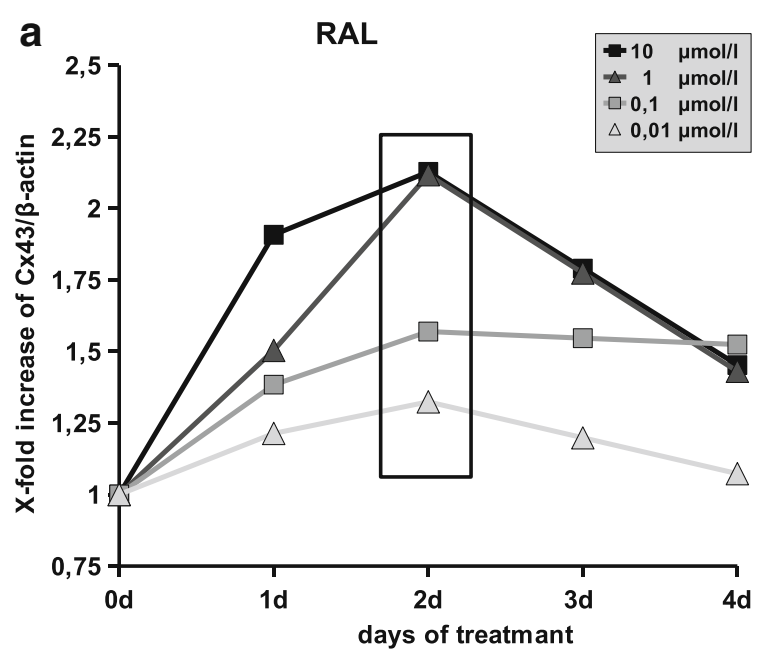

b

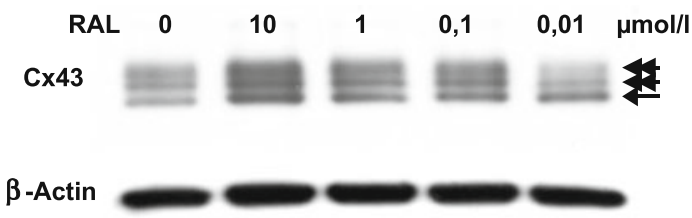

C

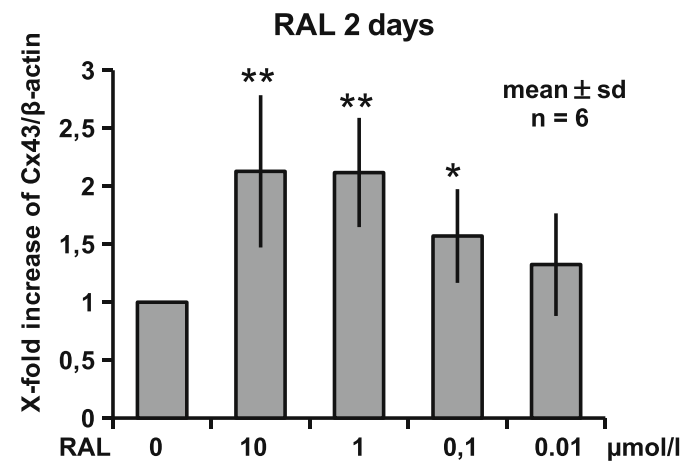

Fig. 3 Time and concentration dependency of RAL effects on Cx43 immunoreactivity in NTera2/D1 cells as revealed by Western blot analysis. a Densitometric evaluation of a series of Western blots, demonstrating that in undifferentiated NTera2/ D1 cells RAL leads to a time- and concentration-dependent increase in $\mathrm{Cx} 43$ immunoreactivity, reaching a maximum at 2 days of treatment. b Representative Western blot of Cx43 immunoreactivity in NTera2/D1 cells, treated for 2 days with RAL at concentrations between 10 and $0.01 \mu \mathrm{mol} / 1$, as depicted by the square in (a). c Diagram of the statistical evaluation of a series of blots as shown in (b), demonstrating a significant upregulation of $\mathrm{Cx} 43$ immunoreactivity by RAL at concentrations of 10,1 , and $0.1 \mu \mathrm{mol} / 1 .{ }^{*} p \leq 0.05 ;{ }^{* *} p \leq 0.01$ 

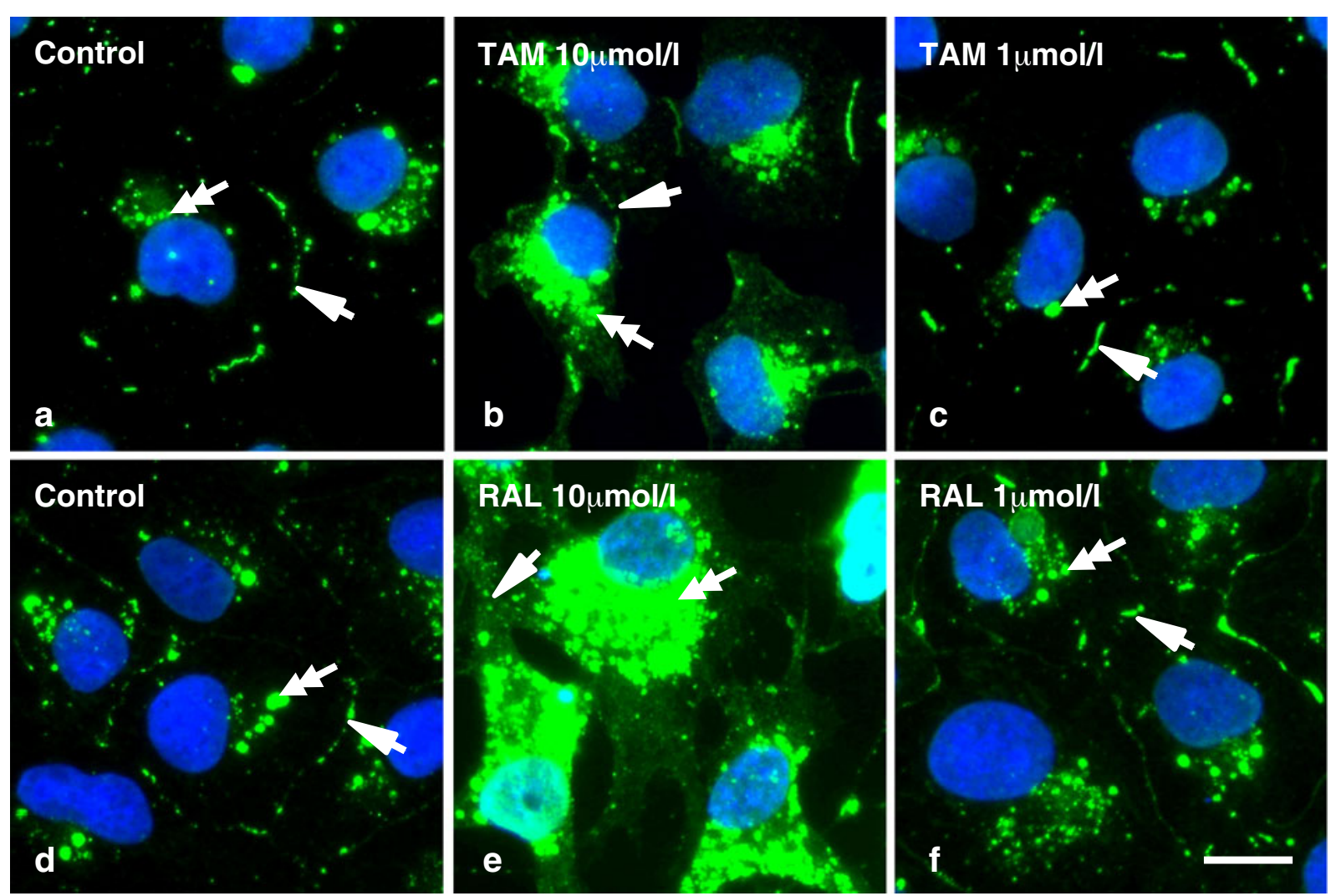

Fig. 4 TAM- and RAL-dependent changes in subcellular distribution of $\mathrm{Cx} 43$, as revealed by immunofluorescent staining. In control cells (a, d), Cx43 immunoreactivity can be detected both at the cell membrane (arrowheads), and in intracellular stores (double arrows). After treatment with $10 \mu \mathrm{mol} / 1$ of either TAM (b), or RAL (e), a distinct increase

almost no impact on RA-induced neuronal differentiation of NTera2/D1 cells. Finally, Western blot analysis for expression of $\beta$ III-Tubulin in NTera2/D1 cells reveals the effects of an early treatment with TAM (Fig. 8i) or RAL (Fig. 8k) on expression of $\beta$ III-Tubulin in NTera2/ D1 cells to be concentration dependent.

\section{Discussion}

Results of the present study demonstrate high-dose treatment with TAM and RAL to elicit a temporary increase of $\mathrm{Cx} 43$ levels and functional GJ coupling in NTera2/D1 cells, and to diminish the RA-dependent downregulation of $\mathrm{Cx} 43$ expression and functional GJ coupling normally observed during neuronal differentiation of these cells. According to this, both in intracellular Cx43 immunoreactivity (double arrows) can be detected, whereas staining at the cell borders (arrowheads) seems to be diminished. At concentrations of $1 \mu \mathrm{mol} / 1$ of either TAM (c), or RAL (f), no gross changes in subcellular distribution of $\mathrm{Cx} 43$ can be observed. Scale bar represents $10 \mu \mathrm{m}$

substances are able to interfere with normal neuronal differentiation when applied during early phases of neuronal determination, whereas already differentiated neurons are not affected. These findings therefore confirm earlier results on the role of $\mathrm{Cx} 43$ and GJ coupling during neuronal differentiation of NTera2/ D1 cells (Bani-Yaghoub et al. 1997, 1999; Boucher and Bennett 2003) and link these effects to the actions of TAM and RAL in these cells.

Effects of TAM on GJ coupling have been earlier demonstrated in non-neural cell types, such as cultured cardiac myocytes (Verrecchia and Hervé 1997), or breast cancer cells (Sáez et al. 2003). Thus, in cultured cardiac myocytes, TAM leads to a reduction in GJ coupling at similar concentrations used in the present study (Verrecchia and Hervé 1997). In contrast, the human breast cancer cell line 

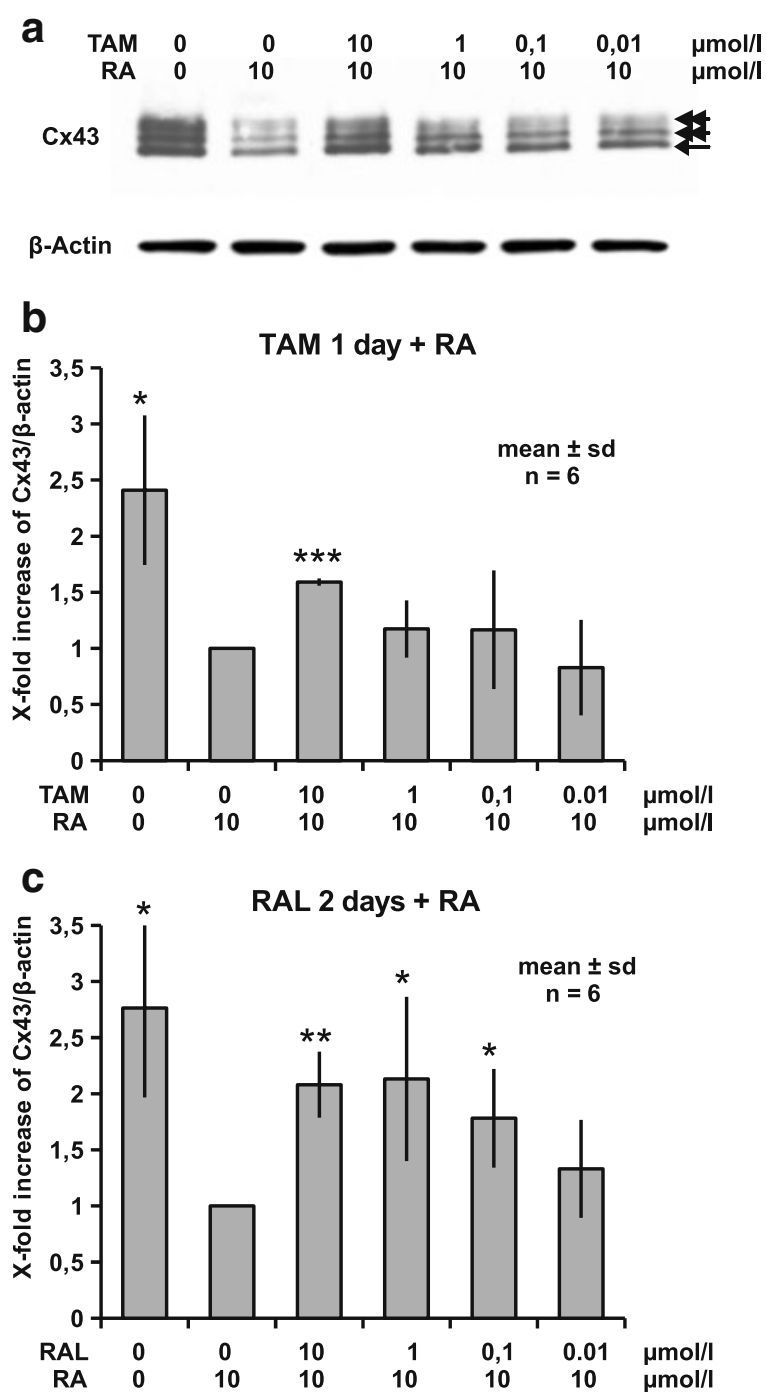

Fig. 5 Western blot analysis, demonstrating that in NTera2/D1 cells, TAM and RAL are able to prevent the effects of RA on Cx43 immunoreactivity in a concentration-dependent manner. a Representative Western blot of Cx43 immunoreactivity in NTera2/D1 cells treated for 1 day with either $10 \mu \mathrm{mol} / 1 \mathrm{TAM}$ or RA alone, or with $10 \mu \mathrm{mol} / \mathrm{l} \mathrm{RA}$ in combination with different concentrations of TAM. b Diagram of the statistical evaluation of a series of blots as shown in (a), demonstrating the prevention of the RA-dependent decrease in Cx43 immunoreactivity by TAM to be highly significant. c Diagram of the statistical evaluation of a series of blots demonstrating the prevention of the RA-dependent decrease in $\mathrm{Cx} 43$ immunoreactivity by RAL to be significant at concentrations of $0.1 \mu \mathrm{mol} / 1$ and higher. ${ }^{*} p \leq 0.05 ;{ }^{*} p \leq 0.01 ; * * * p \leq 0.001$

MCF-7 reacts to a treatment with TAM with an increase in $\mathrm{Cx}$ expression and GJ coupling, being probably associated with the antiproliferative effect of TAM in these cells (Sáez et al. 2003). Obviously both cell types display differential reaction patterns, suggesting also the effects of TAM and RAL on $\mathrm{Cx}$ expression and GJ coupling of the present study, to be cell type specific.

The cell-type-specific actions of TAM and RAL on GJ coupling, suggest specific subcellular mechanisms to be involved. Therefore, in MCF-7 cells concentration dependency of the effects of TAM and RAL on GJ coupling, suggests an estrogen receptor (ER)dependent mechanism (Sáez et al. 2003). However, despite the fact that NTera2/D1 cells express ERs (Chen et al. 2003; Pierson et al. 2005), the comparably high concentrations of TAM and RAL necessary to elicit changes in GJ coupling in NTera2/D1 cells, are contradictory to a receptor-mediated mechanism. Alternatively, the lipophilic nature of both substances could argue for changes in membrane fluidity to modulate intercellular GJ communication in NTera2/ D1 cells (Bennett and Verselis 1992). For TAM such an effect has been already demonstrated in cultured cardiac myocytes, where a reversible inhibition of GJ coupling has been described with an $\mathrm{IC}_{50}$ of around $5 \mu \mathrm{mol} / \mathrm{l}$ (Verrecchia and Hervé 1997).

A mechanism which seems not to be involved in the effects observed in NTera2/D1 cells is $\mathrm{Cx}$ phosphorylation. Functionality of GJ channels consisting of several $\mathrm{Cx}$ types such as $\mathrm{Cx} 43$ is regulated by a step-wise phosphorylation, such that only phosphorylated forms of $\mathrm{Cx} 43$ build functional GJ channels (Solan and Lampe 2009). However, Western blot results of our study do not show such a mechanism in NTera2/D1 cells, since the increase of total $\mathrm{Cx} 43$ protein by TAM and RAL is not accompanied by a disproportional increase of the bands representing the phosphorylated forms of $\mathrm{Cx} 43$. Instead, as revealed by Western blot, the increase in the rate of GJ coupling by TAM and RAL is probably mostly due to an increase in total $\mathrm{Cx} 43$ protein synthesis and a resulting increase in the number of functional GJs at the cell membrane. However, as revealed by immunocytochemistry, not all $\mathrm{Cx} 43$ molecules are integrated into the cell membrane, but also subcellular localization is greatly affected by both TAM and RAL. Thus, Cx43 seems to accumulate in intracellular organelles, such as the Golgi apparatus or the trans Golgi network, as well as in lysosomes. An argument for the latter possibility is the small size of $\mathrm{Cx} 43$ immunoreactive vesicles; however, more detailed studies with organelle- 

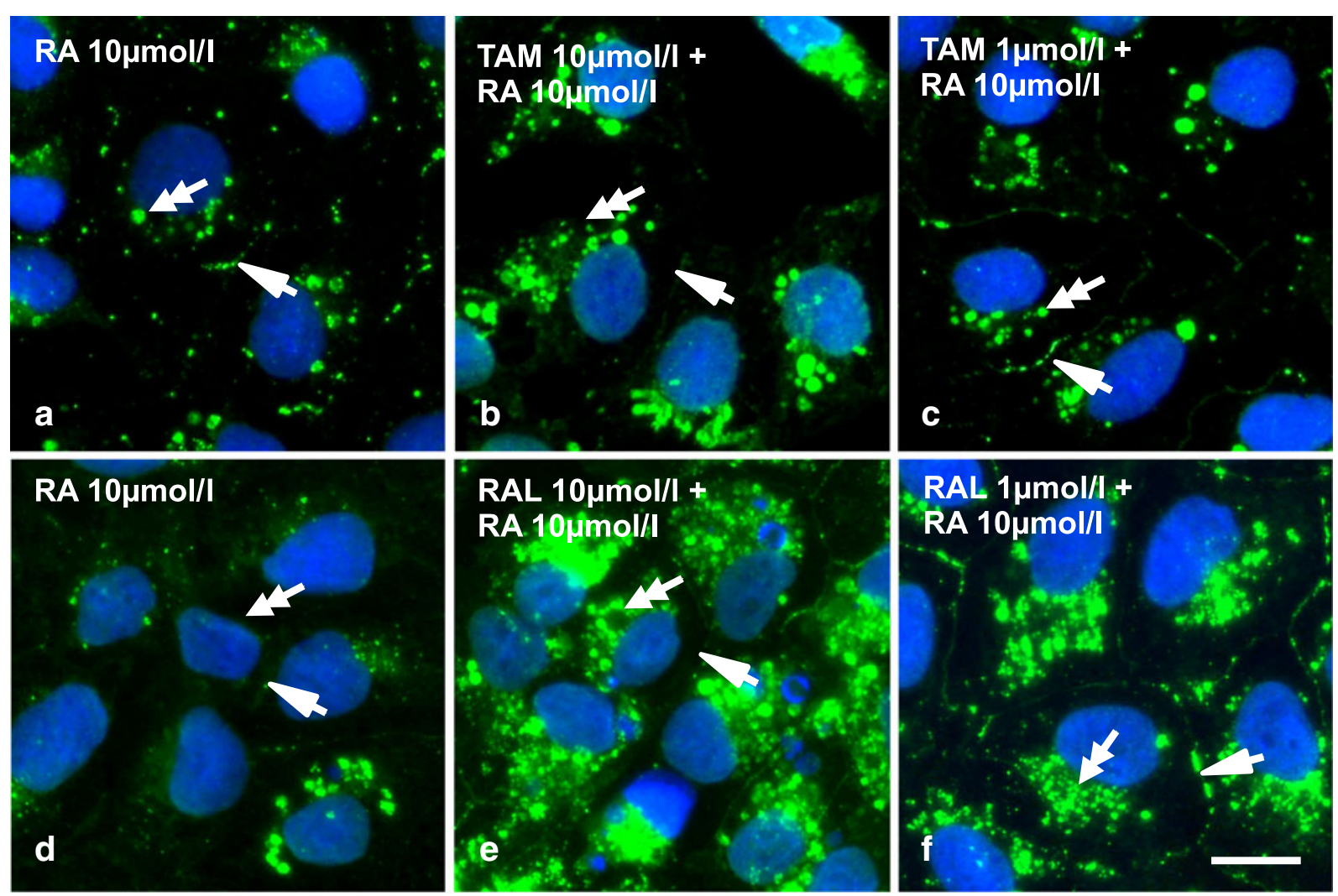

Fig. 6 Subcellular distribution of $\mathrm{Cx} 43$ in NTera2/D1 cells treated with RA alone or in combination with TAM and RAL, as revealed by immunofluorescent staining. In cells treated with $10 \mu \mathrm{mol} / 1$ of RA alone (a, d), weak Cx43 immunoreactivity can be detected both at the cell borders (arrowheads), and in intracellular stores (double arrows). After treatment with $10 \mu \mathrm{mol} / 1$ of either TAM (b), or RAL (e), in combination with $10 \mu \mathrm{mol} / 1 \mathrm{RA}$, a distinct increase in intracellular $\mathrm{Cx} 43$

specific markers will have to be done in order to clarify this point in the future.

Another important point with regard to the effects observed in the present study, is the question whether concentrations of TAM and RAL applied here are within or beyond therapeutically relevant levels. On the first glance the latter seems to be the case, since during long-term TAM treatment, steady state serum concentrations of up to $200 \mathrm{ng} / \mathrm{ml}\left(\sim 5.4 \times 10^{-8} \mathrm{~mol} / \mathrm{l}\right)$ were reported (Jordan 1982; Slee et al. 1988), which are only slightly above the lowest concentrations applied here. For RAL even lower steady-state serum concentrations of $0.5 \mathrm{ng} / \mathrm{ml}$ have been reported (Snyder et al. 2000; Heringa 2003). However, due to their lipophilic properties, serum concentrations of TAM and RAL do not always represent actual levels immunoreactivity (double arrows) can be detected. NTera2/ D1 cells treated with $1 \mu \mathrm{mol} / 1$ of either TAM (c), or RAL (f), together with $10 \mu \mathrm{mol} / \mathrm{l} \mathrm{RA}$, reveal only a slight increase in cytoplasmic Cx43 immunoreactivity (double arrows), and, even more important, a clear recovery of $\mathrm{Cx} 43$ immunoreactive GJ plaques at the cell borders (arrowheads). Scale bar represents $10 \mu \mathrm{m}$

in specific tissues. Thus, in human brain samples, obtained during surgical removal of breast cancer metastases, TAM concentrations of up to $1.5 \mu \mathrm{g}$ per gram of tissue weight were detected (Lien et al. 1991), suggesting that at least local concentrations of TAM would be sufficient to modulate gap junction coupling as it has been described here. In contrast to that, RAL, at least in the rat brain seems not to be locally enriched (Yang et al. 2007). This could explain why RAL despite its obvious in vitro effects has less brain specific side effects in patients. Nevertheless, the highest concentration of $10 \mu \mathrm{mol} / \mathrm{l}$ of TAM and RAL applied here, is obviously exceeding therapeutically relevant concentrations, and suggest the effects observed here to be mostly of relevance for acute toxicity. 

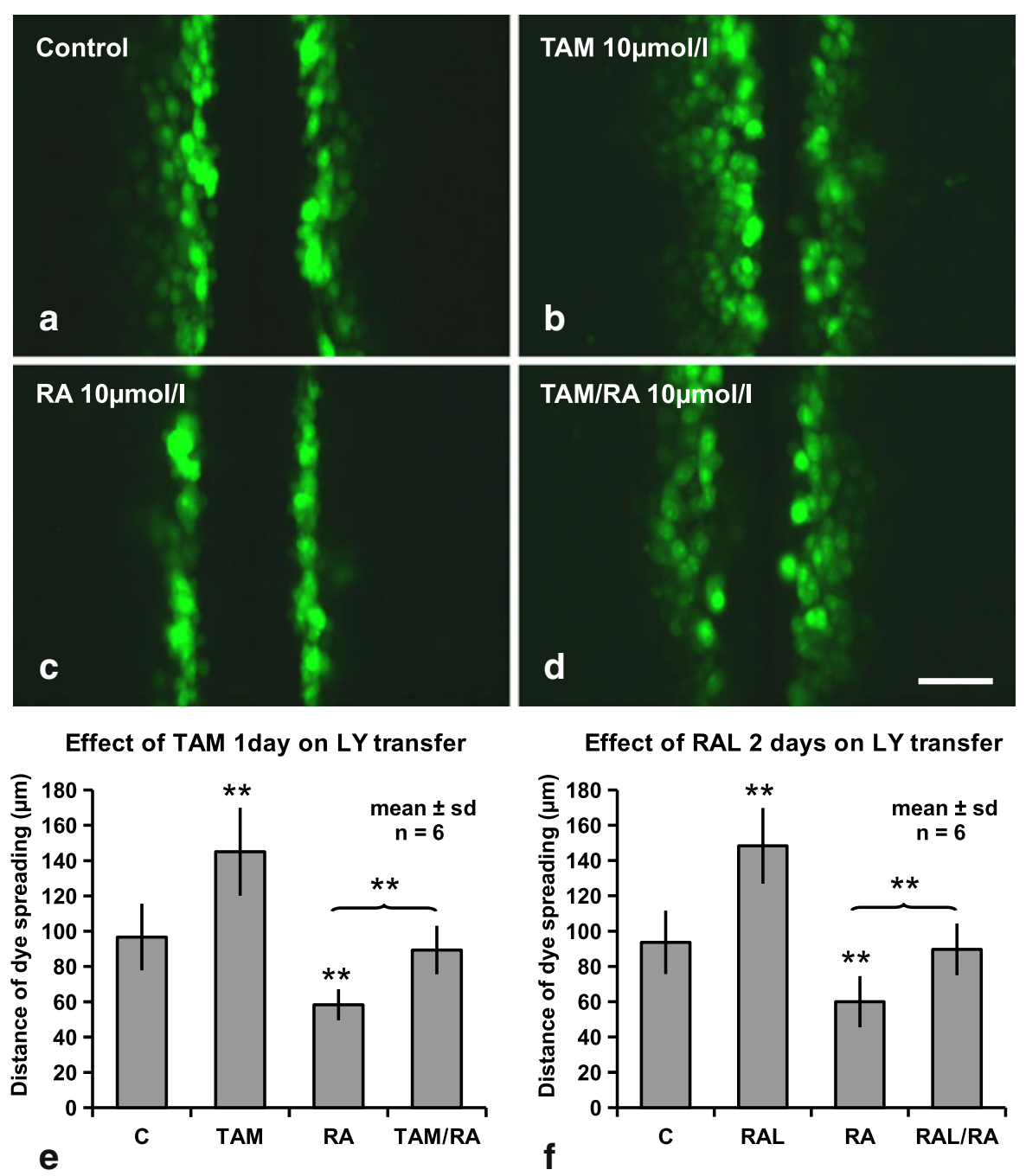

Fig. 7 Effects of TAM or RAL alone or in combination with RA, on spreading of the GJ permeant dye LY between NTera2/ D1 cells, as revealed by scrape loading. In control cultures of NTera2/D1 cells (a, e), spreading of LY to cells not directly adjacent to the scrape, can be readily detected. Treatment with $10 \mu \mathrm{mol} / 1 \mathrm{TAM}(\mathbf{b}, \mathbf{e})$ clearly increases the spreading distance of LY, whereas treatment with $10 \mu \mathrm{mol} / 1 \mathrm{RA}(\mathbf{c}, \mathbf{e})$, diminishes dye spreading between NTera2/D1 cells. Combined treatment of NTera2/D1 cells with $10 \mu \mathrm{mol} / 1$ of each RA and TAM (d, e), is able to restore dye spreading almost to control levels. e

An important finding of the present study is also that TAM and RAL are able to prevent RA-dependent downregulation of $\mathrm{Cx} 43$ on both expressional and functional levels. Together with earlier findings that RA-dependent neuronal differentiation of NTera2/D1 cells is associated with a reduction of $\mathrm{Cx} 43$ expression and GJ coupling (Bani-Yaghoub et al. 1997, 1999; Boucher and Bennett 2003), this suggests TAM

\section{Effect of RAL 2 days on LY transfer}

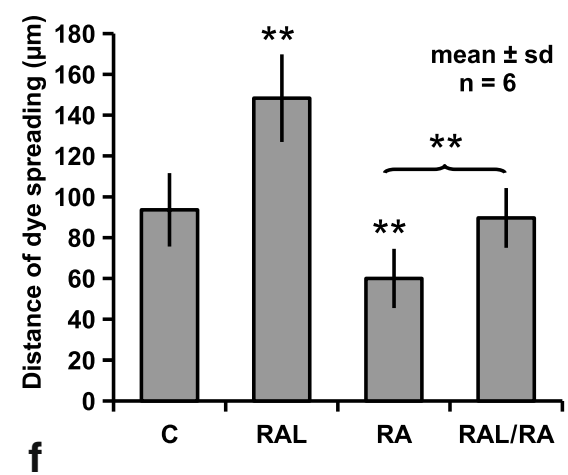

Statistical evaluation of the spreading distances at 10 randomly chosen points in each of four scrapes set in six independent experiments, reveals the differences described above to be highly significant. f Also a treatment of NTera2/D1 cells with $10 \mu \mathrm{mol} / 1$ RAL, leads to an increased dye-spreading rate as compared to normal control cells, whereas a combined treatment with $10 \mu \mathrm{mol} / \mathrm{l}$ of each RA and RAL, leads to a restored dye spreading rate between NTera2/D1 cells, as compared to cells treated with $10 \mu \mathrm{mol} / 1 \mathrm{RA}$ alone. Scale bar represents $100 \mu \mathrm{m}$

and RAL to interfere also with neuronal differentiation of these cells. According to this, the present study demonstrates that both TAM and RAL affect neuronal differentiation of NTera2/D1 cells during the early phase of RA-dependent differentiation, whereas application during the late phase of RA treatment has no such effect. Changes in intercellular communication by GJ between NTera2/D1 cells elicited by TAM and 

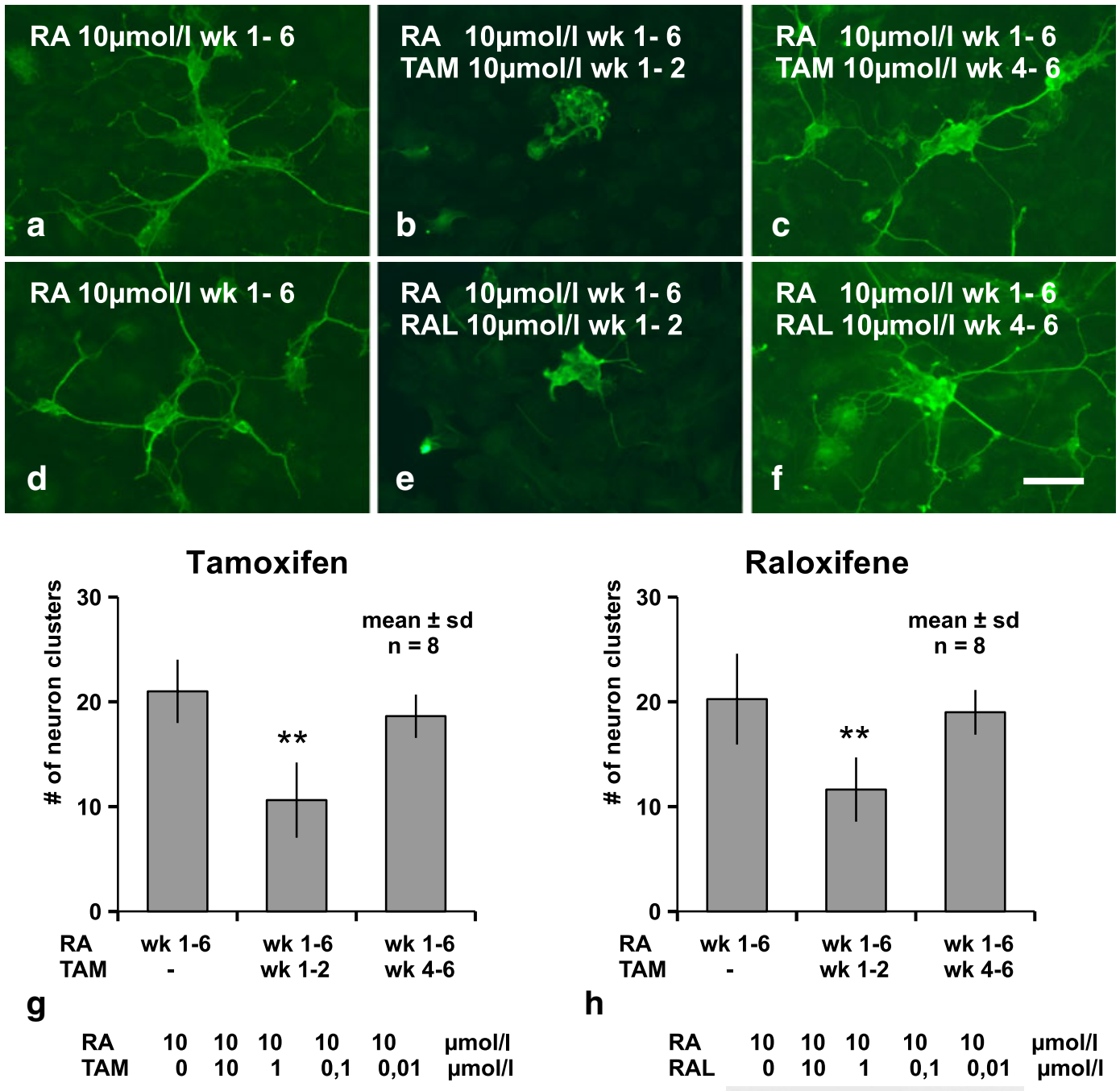

$\beta 3-T u b u l i n$

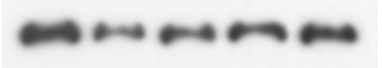

$\beta-A c t i n$

$\beta$-Actin

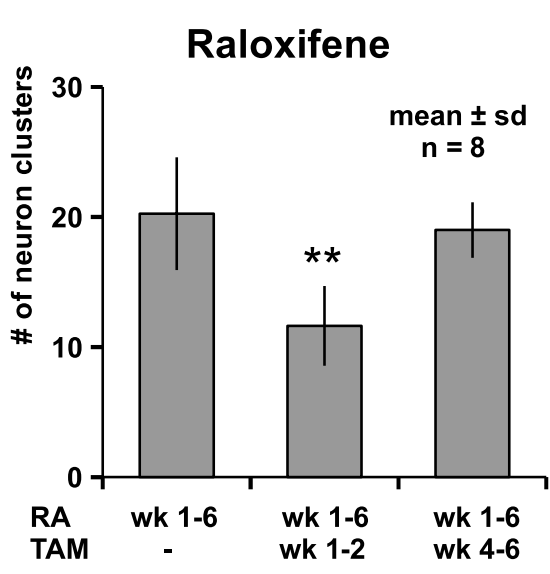

h

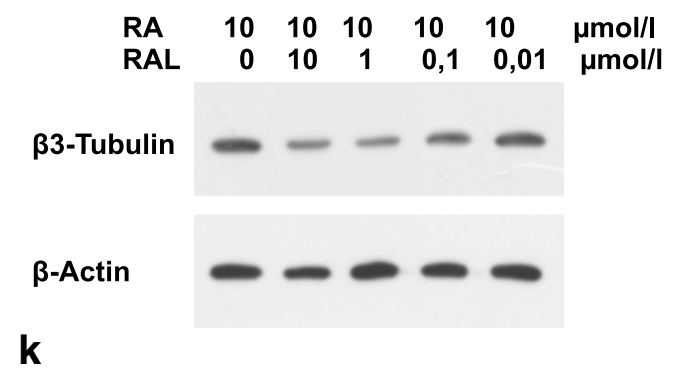

Fig. 8 Effects of TAM or RAL on RA-dependent neuronal differentiation of NTera2/D1 cells, revealed by the marker

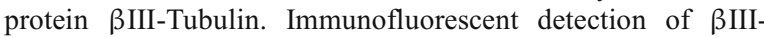
Tubulin in NTera2/D1 cells differentiated for 6 weeks by $10 \mu \mathrm{mol} / 1 \mathrm{RA}(\mathbf{a}, \mathbf{d})$ reveals strongly stained neuronal clusters which are intensely interconnected by neuron processes. Cultures treated with $10 \mu \mathrm{mol} / 1$ of either TAM (b) or RAL (e) during weeks 1 and 2 of a 6 weeks RA-dependent neuronal differentiation schedule, reveal reduced numbers of $\beta$ III-Tubulin positive neuron clusters, as well as decreased neuronal process formation. In contrast, cultures treated with $10 \mu \mathrm{mol} / 1$ of either

TAM (c) or RAL (f) during weeks 5 and 6, are almost indistinguishable from the control cultures treated with RA alone. $\mathbf{g}, \mathbf{h}$ Statistical evaluation of a set of eight independent experiments as shown in $\mathbf{a}-\mathbf{f}$, revealing the effects of TAM (g) and RAL (h), to be highly significant. i, k Western blot analysis for $\beta$ III-Tubulin in NTera2/D1 cells treated with different concentrations of either TAM (i) or RAL (k) during the first 2 weeks of a 6 weeks RA-dependent differentiation schedule. Actions of both substances are obviously concentration dependent. Scale bar represents $25 \mu \mathrm{m}$ 
RAL seems therefore not to affect differentiated neurons in the mature brain, however, might be of importance for an understanding of effects of TAM and RAL during early nervous system formation in the embryo.

In several brain lesion paradigms, such as middle cerebral artery occlusion (Mehta et al. 2003) or permanent focal ischemia (Kimelberg et al. 2003), TAM has been shown to act neuroprotective and upregulation of GJ intercellular communication plays a role for these effects (Hossain et al. 1994; Nakase et al. 2003). This seems on the first glance to be contradictory to our findings. However, as discussed before, the effects described here are observed only during early phases of neuronal differentiation, whereas in later phases, TAM and RAL are no longer able to impair neuronal differentiation and functioning. Whether under these circumstances TAM or RAL act in a protective manner has not been addressed by the present study and therefore remains to be clarified in the future.

Acknowledgments We would like to thank the medical faculty of the University of Göttingen (UMG) for persistent and reliable support of our work.

Open Access This article is distributed under the terms of the Creative Commons Attribution Noncommercial License which permits any noncommercial use, distribution, and reproduction in any medium, provided the original author(s) and source are credited.

\section{References}

Andrews PW. Retinoic acid induces neuronal differentiation of a cloned human embryonal carcinoma cell line in vitro. Dev Biol. 1984;103:285-93.

Bani-Yaghoub M, Bechberger JF, Naus CC. Reduction of connexin43 expression and dye-coupling during neuronal differentiation of human NTera2/clone D1 cells. J Neurosci Res. 1997;49:19-31.

Bani-Yaghoub M, Bechberger JF, Underhill TM, Naus CC. The effects of gap junction blockage on neuronal differentiation of human NTera2/clone D1 cells. Exp Neurol. 1999;156:16-32.

Bennett MV, Verselis VK. Biophysics of gap junctions. Semin Cell Biol. 1992;3:29-47.

Berger JC, Clericuzio CL. Pierre Robin sequence associated with first trimester fetal tamoxifen exposure. Am J Med Genet A. 2008;146A:2141-4.

Boucher S, Bennett SA. Differential connexin expression, gap junction intercellular coupling, and hemichannel formation in
NT2/D1 human neural progenitors and terminally differentiated hNT neurons. J Neurosci Res. 2003;72:393-404.

Bruzzone R, Dermietzel R. Structure and function of gap junctions in the developing brain. Cell Tissue Res. 2006;326:239-48. Review.

Byrd RA, Francis PC. The selective estrogen receptor modulator, raloxifene: segment II studies in rats and rabbits. Reprod Toxicol. 1998;12:261-70.

Chen G, Shinka T, Kinoshita K, Yan HT, Iwamoto T, Nakahori Y. Roles of estrogen receptor $\alpha(E R \alpha)$ in the regulation of the human Müllerian inhibitory substance (MIS) promoter. J Med Invest. 2003;50:192-8.

Collins B, Mackenzie J, Stewart A, Bielajew C, Verma S. Cognitive effects of hormonal therapy in early stage breast cancer patients: a prospective study. Psychooncology. 2009;18:811-21.

Cunha GR, Taguchi O, Namikawa R, Nishizuka Y, Robboy SJ. Teratogenic effects of clomiphene, tamoxifen, and diethylstilbestrol on the developing human female genital tract. Hum Pathol. 1987;18:1132-43.

da Rocha AB, Mans DR, Bernard EA, Ruschel C, Logullo AF, Wetmore LA, et al. Tamoxifen inhibits particulateassociated protein kinase $\mathrm{C}$ activity, and sensitises cultured human glioblastoma cells not to etoposide but to $\gamma$ radiation and BCNU. Eur J Cancer. 1999;35:833-9.

Eberling JL, Wu C, Tong-Turnbeaugh R, Jagust WJ. Estrogenand tamoxifen-associated effects on brain structure and function. Neuroimage. 2004;21:364-71.

Fisher B, Costantino JP, Redmond CK, Fisher ER, Wickerham DL, Cronin WM. Endometrial cancer in tamoxifen-treated breast cancer patients: findings from the National Surgical Adjuvant Breast and Bowel Project (NSABP) B-14. J Natl Cancer Inst. 1994;86:527-37.

Guthrie SC, Gilula NB. Gap junctional communication and development. Trends Neurosci. 1989;12:12-6.

Heringa M. Review on raloxifene: profile of a selective estrogen receptor modulator. Int J Clin Pharmacol Ther. 2003;41:331-45.

Hill EJ, Woehrling EK, Prince M, Coleman MD. Differentiating human NT2/D1 neurospheres as a versatile in vitro 3D model system for developmental neurotoxicity testing. Toxicology. 2008;249:243-50.

Hossain MZ, Peeling J, Sutherland GR, Hertzberg EL, Nagy JI. Ischemia-induced cellular redistribution of the astrocytic gap junctional protein connexin43 in rat brain. Brain Res. 1994;652:311-22.

Jordan VC. Metabolites of tamoxifen in animals and man: identification, pharmacology, and significance. Breast Cancer Res Treat. 1982;2:123-38.

Kimelberg HK, Jin Y, Charniga C, Feustel PJ. Neuroprotective activity of tamoxifen in permanent focal ischemia. J Neurosurg. 2003;99:138-42.

Lien EA, Wester K, Lønning PE, Solheim E, Ueland PM. Distribution of tamoxifen and metabolites into brain tissue and brain metastases in breast cancer patients. Br J Cancer. 1991;63:641-5.

Mehta SH, Dhandapani KM, De Sevilla LM, Webb RC, Mahesh VB, Brann DW. Tamoxifen, a selective estrogen receptor modulator, reduces ischemic damage caused by middle cerebral artery occlusion in the ovariectomized female rat. Neuroendocrinology. 2003;77:44-50. 
Meșe G, Richard G, White TW. Gap junctions: basic structure and function. J Invest Dermatol. 2007;127:2516-24.

Nakase T, Fushiki S, Naus CC. Astrocytic gap junctions composed of connexin 43 reduce apoptotic neuronal damage in cerebral ischemia. Stroke. 2003;34:1987-93.

Nobakht M, Najafzadeh N, Kordestani Shargh B. Effects of tamoxifen on morphological and ultrastructural aspects of developing hippocampus of rat. Iran Biomed J. 2009; 13:171-7.

Palmer JL, Trotter T, Joy AA, Carlson LE. Cognitive effects of Tamoxifen in pre-menopausal women with breast cancer compared to healthy controls. J Cancer Surviv. 2008;2:275-82.

Pierson RC, Lyons AM, Greenfield Jr LJ. Gonadal steroids regulate $\mathrm{GABA}_{\mathrm{A}}$ receptor subunit mRNA expression in NT2-N neurons. Brain Res Mol Brain Res. 2005;138:105-15.

Pleasure SJ, Lee VM. NTera2 cells: a human cell line which displays characteristics expected of a human committed neuronal progenitor cell. J Neurosci Res. 1993;35:585-602.

Reginster JY, Devogelaer JP. Raloxifene reduces fractures in postmenopausal women with osteoporosis. Clin Orthop Relat Res. 2006;443:48-54.

Revel JP. Gap junctions in development. Dev Biol. 1986;3:191-204.

Sáez CG, Velásquez L, Montoya M, Eugenín E, Alvarez MG. Increased gap junctional intercellular communication is directly related to the anti-tumor effect of all-trans-retinoic acid plus tamoxifen in a human mammary cancer cell line. J Cell Biochem. 2003;89:450-61.

Slee PH, De Vos D, Chapman D, Stevenson D. The bioavailability of Tamoplex (tamoxifen). Part 3. A steady-state study in breast cancer patients. Pharm Weekbl Sci. 1988;10:22-5.

Snyder KR, Sparano N, Malinowski JM. Raloxifene hydrochloride. Am J Health Syst Pharm. 2000;57:1669-75.
Söhl G, Willecke K. Gap junctions and the connexin protein family. Cardiovasc Res. 2004;62:228-32.

Solan JL, Lampe PD. Connexin43 phosphorylation: structural changes and biological effects. Biochem J. 2009;419:261-72.

Sutor B, Hagerty T. Involvement of gap junctions in the development of the neocortex. Biochim Biophys Acta. 2005;1719:59-68.

Verrecchia F, Hervé J. Reversible inhibition of gap junctional communication by tamoxifen in cultured cardiac myocytes. Pflüger's Arch. 1997;434:113-6.

Wei $\mathrm{CJ}, \mathrm{Xu} \mathrm{X}$, Lo CW. Connexins and cell signaling in development and disease. Annu Rev Cell Dev Biol. 2004;20:811-38.

Wickerham DL, Costantino JP, Vogel VG, Cronin WM, Cecchini RS, Ford LG, et al. The use of tamoxifen and raloxifene for the prevention of breast cancer. Recent Results Cancer Res. 2009;181:113-9.

Williams GM, Iatropoulos MJ, Djordjevic MV, Kaltenberg OP. The triphenylethylene drug tamoxifen is a strong liver carcinogen in the rat. Carcinogenesis. 1993;14:315-7.

Woehrling EK, Hill EJ, Coleman MD. Development of a neurotoxicity test-system, using human post-mitotic, astrocytic and neuronal cell lines in co-culture. Toxicol In Vitro. 2007;21:1241-6.

Woehrling EK, Hill EJ, Coleman MD. Evaluation of the importance of astrocytes when screening for acute toxicity in neuronal cell systems. Neurotox Res. 2010;17:103-13.

Yaffe K, Krueger K, Cummings SR, Blackwell T, Henderson VW, Sarkar S, et al. Effect of raloxifene on prevention of dementia and cognitive impairment in older women: the Multiple Outcomes of Raloxifene Evaluation (MORE) randomized trial. Am J Psychiatry. 2005;162:683-90.

Yang Z, He X, Zhang Y. The determination of raloxifene in rat tissue using HPLC. Biomed Chromatogr. 2007;21:229-33. 